Artigo de Revisão

Hegemonia - Revista Eletrônica do Programa de Mestrado em Direitos Humanos,

Cidadania e Violência/Ciência Política do Centro Universitário Unieuro

ISSN: 1809-1261

UNIEURO, Brasília, número 27 (Especial), 2019, pp. 7-27.

Recebido em: 18/9/2018

Avaliado em: 24/10/2018

Aprovado em: 9/11/2018

\title{
USO DE FOTOTERAPIA PARA CICATRIZAÇÃO DE FERIDAS DE PÉS DIABÉTICOS
}

\author{
Franciéle de Matos da Silva ${ }^{1}$, Lorena de Sousa Moreira ${ }^{2}$, Mayla dos Santos Silva ${ }^{3}$, \\ Wellington Rodrigues ${ }^{4}$ e Suélia de Siqueira Rodrigues Fleury Rosa ${ }^{5}$
}

Resumo: A fototerapia é uma modalidade terapêutica que possui atividade antiinflamatória, que promove a regeneração e reparo dos tecidos conjuntivos através da estimulação da síntese e proliferação da matriz extracelular. O objetivo desta revisão sistemática é sintetizar os estudos científicos já realizados, que abordam o uso de LEDs e látex biomembrana(BML) para o tratamento de feridas do pé diabético. Métodos: Revisão da literatura científica utilizando as bases de dados Scielo, Lilacs, Pubmed, Bireme, Medline, foram considerados artigos entre 2009 a 2018, com os seguintes descritores: "diabetes mellitus","pé diabético", "processo cicatricial", "RAPHA". Resultado: Foram encontrados 637 artigos dos descritores selecionados, apena 48 foram potencialmente consideráveis para oestudo. Publicações duplicadas foram removidas e artigos que estavam presentes em mais de um banco de dados. O LED é um excelente tratamento para feridas do pé diabético e, quando associado ao MLL, seus efeitos têm sido potencializados.Conclusão: Nesta revisão, verificamos a eficácia da fototerapia nas feridas do pé diabético. O LED é mais barato que o laser, por isso é usado com o intuito de popularizar o tratamento e, quando associado à membrana, torna seus efeitos mais poderosos.

Palavras-Chaves: Diabetes, Pé diabético, Formação tecidual, LED.

Abstract: Phototherapy is a therapeutic modality that has anti-inflammatory activity, which promotes the regeneration and repair of connective tissues through the stimulation of the synthesis and proliferation of the extracellular matrix. Objective: The purpose of this systematic review is to synthesize the scientific studies already carried out, which address the use of LEDs and biomembrane latex (BML), for the treatment of diabetic foot wounds. Methods: Review of scientific literature using the databases Scielo, Lilacs, PubMed, Bireme, Medline, were considered articles between 2009 to 2018, with the

\footnotetext{
1 Mestranda em Engenharia Biomédica pelo Programa de Pós-Graduação em Engenharia Biomédica, da Universidade de Brasília.

2 Mestranda em Engenharia Biomédica pelo Programa de Pós-Graduação em Engenharia Biomédica, da Universidade de Brasília.

3 Mestranda em Engenharia Biomédica pelo Programa de Pós-Graduação em Engenharia Biomédica, da Universidade de Brasília.

${ }^{4}$ Mestrando em Engenharia Biomédica pelo Programa de Pós-Graduação em Engenharia Biomédica, da Universidade de Brasília.

${ }^{5}$ Doutora em Engenharia Eletrônica-Biomédica e docente da Universidade de Brasília
} 
Artigo de Revisão

Hegemonia - Revista Eletrônica do Programa de Mestrado em Direitos Humanos,

Cidadania e Violência/Ciência Política do Centro Universitário Unieuro

ISSN: 1809-1261

UNIEURO, Brasília, número 27 (Especial), 2019, pp. 7-27.

following descriptors: "diabetes mellitus", "diabetic foot", "tissue healing process", "phototherapy" , "RAPHA". Results: There were 637 articles of the selected descriptors found, only 48 were potentially considerable for the study. Duplicates publications were removed and articles that were present in more than one database. The LED is an excellent treatment for diabetic foot wounds and, when associated with MLL, its effects have been potentiated. Conclusion: In this review, we verified the effectiveness of phototherapy in diabetic foot wounds. LED is cheaper than laser, so it is used for the purpose of popularizing the treatment and, when associated with the membrane, makes its effects more powerful.

Keyword: Diabetes, Diabetic foot, Tissue formation, LED.

\section{INTRODUÇÃO}

As primeiras citações relacionadas a feridas, têm origem em (1536 a.C.), onde consiste em um dos tratamentos médicos mais antigos e conhecidos da história. O papiro de Ebers que atualmente se encontra em exibição na biblioteca da Universidade de Leipzig, na Alemanha, preservou o maior registro da medicina Egípcia Antiga, contendo mais de 700 fórmulas de remédios para diversos distúrbios, dentre eles para o tratamento de feridas [1].

Diante da revolução científica, o processo de cicatrização com tratamentos de feridas encontra-se em desafio que tenha maior eficácia para os portadores de Diabetes Mellitus (DM).

Os pacientes diabéticos são definidos como crônicos, que há falhas no mecanismo de cicatrização, contudo afetando a cascata de cicatrização caracterizada por uma resposta débil mais proliferativa da lesão do que exsudativa [2].

Uma das complicações dos portadores de DM é a ulceras de pé diabético (UPD) conhecido popularmente como "pé de diabético", tal complicação afeta cerca de $50 \%$ dos pacientes diabéticos, desses 85\% chegam a amputar o membro [3].

A Organização Mundial da Saúde (OMS) define pé diabético, como infecção, ulceração e destruição dos tecidos profundo dos pés, e está associada a anormalidades neurológicas e vários graus de doença vascular periférica, nos membros inferiores de pacientes com DM. [4].

Perante tal morbidade e ineficácia dos tratamentos atuais, há de se ressaltar a evolução científica de tratamentos inéditos como a fototerapia, com a utilização de laser e LEDs [5]. 
Artigo de Revisão

Hegemonia - Revista Eletrônica do Programa de Mestrado em Direitos Humanos,

Cidadania e Violência/Ciência Política do Centro Universitário Unieuro

ISSN: 1809-1261

UNIEURO, Brasília, número 27 (Especial), 2019, pp. 7-27.

Para atingir êxito no tratamento de feridas com complicações na cicatrização é primordial o conhecimento dos fatores que interferem neste processo. Estes devem estar aliados às intervenções apropriadas, como a fototerapia, assegurando a escolha mais eficaz para o tratamento de feridas em pacientes com diabetes mellitus [6].

Uma nova tecnologia está em desenvolvimento, um novo tratamento com altíssima potencialidade para tratar o pé diabético, induzindo assim a cicatrização das úlceras de pés diabéticos - UPD está vigorando.

Um Equipamento Médico Portátil de Neoformação Tecidual - RAPHA, usado juntamente com a Biomembrana de látex (BML) derivada da seringueira (Hevea brasiliensis) um biomaterial de baixo custo, ambos possuem propriedades cicatrizantes com alta capacidade de estabelecer as funçõesangiogênicastem sido utilizada apresentando excelentes resultados.

\section{PÉS DIABÉTICOS}

O diabetes mellitus (DM) é um dos grandes problemas de saúde pública, com altos índices de morbidade e mortalidade, as complicações crônicas têm se tornado cada vez mais comum causando amputações não traumáticas [7].

DM é relacionado à cicatrização deficitária, devido às lesões vasculares (hipóxia) e às alterações nas células fagocitárias, que favorecem a instalação de infecções; ao estreitamento da perfusão sanguínea (vasculopatia); à neuropatia, redução da sensibilidade, por causa da diminuição de estímulos da inflamação liberados por terminações nervosas[8].

Os pés são os mais afetados pela falta de sensibilidade. Pacientes acometidos por essa doença perde os mecanismos de proteção à dor e desenvolve feridas nos pés. O fluxo sanguíneo e prejudicado e as feridas torna-se maior, as lesões pode ser porta de entrada para infecções graves se não tratada em estágios iniciais facilitando assim as úlceras[7].

A ulceração do pé diabético configura uma adversidade médica, social e econômica em todo o mundo. Conforme descrito por Duarte e Gonçalves, o indício de um paciente diabético desenvolver úlceras nos pés pode alcançar 25\%. Essas lesões são a maioria dos casos de internçãoes nos hospitais quando comparadas às outras complicações originadas pela diabetes [9]. 
Artigo de Revisão

Hegemonia - Revista Eletrônica do Programa de Mestrado em Direitos Humanos,

Cidadania e Violência/Ciência Política do Centro Universitário Unieuro

ISSN: 1809-1261

UNIEURO, Brasília, número 27 (Especial), 2019, pp. 7-27.

Pessos com DM, as úlceras caracterizam-se por lesões cutâneas com perda do epitélio, que se estendem até a derme ou atravessa e chegando aos tecidos mais profundos, podendo atingir ossos e musculos [10]

O tratamento depende do grau do dano causado ao membro, atualmente existem diversas opções para o tratamento das lesões, vários tipos de curativos, fototerapia, oxigenoterapia hiperbárica, uso de derme humana cultivada (Dermagraft), fatores de crescimentos locais e em casos extremos amputação do membro [7].

O novo tratamento e um equipamento com uma matriz de diodos emissores de luz (LED) sistema indutor de neoformação Tecidual associado com o látex natural que também possui propriedades que favorece a regeneração tecidual [7].

\section{DINÂMICA DO PROCESSO DE REPARO TECIDUAL}

A cicatrização é o processo de reparação no qual ocorre a substituição de um tecido danificado por tecido conjuntivo vascularizado, seja a lesão traumática ou necrótica[8].

O processo de cicatrização tecidual é classificado em fases sobrepostas: hemostasia, fase inflamatória, formação do tecido de granulação e remodelação $[11,12]$.

A fase hemostasia depende da atividade plaquetária e da cascata de coagulação, é iniciada em seguida ao surgimento da lesão. Com a ruptura dos vasos sanguíneos e o extravasamento de seus componentes há estimulação dos constituintes vasoativos a fim de propiciar uma vasoconstrição imediata, tendo em vista atenuar a perda sanguínea. Há agregação plaquetária e coagulação com a finalidade de reduzir essa perda e proteger o organismo da atuação de agentes externos [13].

As plaquetas ativadas tem o potencial de liberar grânulos que contém citocinas, hormônios e quimiocinas, atraindo células inflamatórias para o local da lesão [14]. Alguns pequenos vasos se dilatam permitindo o influxo de leucócitos, células vermelhas sanguíneas e proteínas plasmáticas. A fibrina polimerizada formar um arcabouço que serve para a infiltração de células que são chave para as subsequentes fases do reparo [14].

Há a formação de uma matriz provisória para que ocorra a migração celular exacerbada por meio das vênulas e extravasamento de componentes séricos, anticorpos e proteínas pelos capilares. Essa matriz atua como reservatório de citocinas e fatores de crescimento que serão liberados ao longo das próximas fases da cicatrização. Nessa fase 
Artigo de Revisão

Hegemonia - Revista Eletrônica do Programa de Mestrado em Direitos Humanos,

Cidadania e Violência/Ciência Política do Centro Universitário Unieuro

ISSN: 1809-1261

UNIEURO, Brasília, número 27 (Especial), 2019, pp. 7-27.

tem a presença de células inflamatórias no tecido cicatricial, caracterizando a fase inflamatória [15].

A fase de inflamação é marcada pela entrada de neutrófilos, macrófagos e linfócitos no local da lesão. Os neutrófilos são as primeiras células no local, chegando asprimeiras 24 horas, eo resultado de sua apoptose atrai os macrófagos, que, juntamente com os linfócitos, aparecem na ferida. Os macrófagos permanecem na lesão até o final da fase de inflamação e são consideradas as células chave dessa importante fase do reparo $[13,14]$. A inibição ou prolongamento da fase de inflamação pode resultar em um reparo impróprio, podendo levar à ferida crônica, diminuição da velocidade de cura e, eventualmente, mais cicatriz [14].

A partir disso, ocorre a reparação do tecido conjuntivo e do epitélio por meio da proliferação endotelial e de fibroblastos. Há a formação do tecido de granulação com o depósito de matriz extracelular. O tecido de granulação é originado cerca de quatro dias após a lesão. Ao serem reparados o fluxo sanguíneo e a oxigenação acontece a epitelização que acarreta no fechamento da superfície da lesão [12].

A fase de reparo ou proliferação envolve o brotamento de capilares, a migração e proliferação dos fibroblastos e produção de colágeno imaturo (tipo III) para preencher os defeitos deixados ao longo do debridamento da ferida [14]. Essa proliferação fibroblástica e de capilares dá origem ao tecido de granulação, um tipo de tecido especializado rico em fibronectina e colágeno que é o ponto de referência do reparo tecidual e é predominante na cicatrização por segunda intenção [13,14].

Por fim, sucede a remodelação que é a última fase do processo de reparação tecidual. Essa fase tem durabilidade de meses, e acontece no colágeno e na matriz. Haverá uma diminuição na concentração celular e um aumento da síntese e produção de colágeno. Essa etapa é fundamental para elevar a força de tensão e reduzir o tamanho da cicatriz e do eritema [12]. Com o desenvolvimento do processo de cicatrização, evidencia-se o depósito de colágeno, verificando a apoptose de fibroblastos e células endoteliais; dessa forma, estabelecendo o tecido cicatricial [13].

\section{USO DA FOTOTERAPIA}

Atualmente, a grande aplicabilidade da energia luminosa de forma científica e tecnológica se deve ao raio laser. A origem do laser e discutida. Albert Einstein desenvolveu a teoria 
Artigo de Revisão

Hegemonia - Revista Eletrônica do Programa de Mestrado em Direitos Humanos,

Cidadania e Violência/Ciência Política do Centro Universitário Unieuro

ISSN: 1809-1261

UNIEURO, Brasília, número 27 (Especial), 2019, pp. 7-27.

com a publicação de seu tratado Zur Quantum Theorie der Stralung e foram Schawlaw e Townes os autores dos princípios pelos quais todos os lasers operam. Em 1960 Theodore Maiman conseguiu, pela primeira vez, a emissão estimulada de radiação pela excitação de uma roda de rubi ( $\lambda 1694 \mathrm{~nm}$ ) com pulsos luminosos intensos, gerando assim o primeiro raio laser com emissão no espectro vermelho [14,15]. A palavra laser significa Light AmplificationbyStimulatedEmissionofRadiation, (em português, Amplificação de Luz por Emissão Estimulada) [15].

A geração do raio laser acontece dentro de uma câmara onde suas extremidades estão posicionadas dois espelhos cuja diferença da capacidade de reflexão permite a saída de radiação de dentro da cavidade, formando, assim, o raio laser [16,17].

O laser é uma forma de radiação não ionizante, altamente concentrada, a luz laser consiste em ondas que possuem um comprimento específico, que é a distância entre dois pontoscorrespondentes (picos ou vales) da onda sobre o eixo horizontal.

A frequência é a quantidade total de ondas que passam por um determinado ponto durante o período de um segundo. Logo, quanto menor o comprimento de onda $(\lambda)$, maior será a frequência do raio laser. À medida que o comprimento de onda diminui, a energia aumenta. Assim, lasers com menores comprimentos de onda são mais energéticos. Para o olho humano, comprimento de onda significa cor e apenas a porção do espectro das radiações que se situa entre 400 e 700 nm é visível ao olho humano [16].

A luz laser apresenta como características a coerência, colimação, monocromaticidade, polarização e eficiência. Entretanto, a utilização desse tipo de energia deve levar em consideração importantes parâmetros como potência, densidade de potência, densidade de energia ou fluência, dose, diâmetro do raio, modo de emissão e sistemas e modos de entrega de forma a se potencializar os efeitos benéficos deste tipo de terapia [16].

A potência do laser diz respeito à taxa de entrega de energia em joules por segundo e é medida normalmente em watts, da mesma forma que a luz doméstica convencional. A densidade de potência ou irradiância é a concentração fotônica em uma dada unidade de área e é descrita em watts por centímetro quadrado. Quando o raio laser é focado em determinado local, ocorre a concentração de energia nesse ponto, o que se relaciona diretamente ao comprimento da onda do raio e à distancia da lente ao foco. $\mathrm{O}$ aumento da distância focal implica no aumento do comprimento do raio, diminuindo a densidade da energia aplicada $[16,17]$ 
Artigo de Revisão

Hegemonia - Revista Eletrônica do Programa de Mestrado em Direitos Humanos,

Cidadania e Violência/Ciência Política do Centro Universitário Unieuro

ISSN: 1809-1261

UNIEURO, Brasília, número 27 (Especial), 2019, pp. 7-27.

A absorção do laser pelos tecidos pode resultar em quatro processos: fotoquímico, fototérmico, fotomecânico e fotoelétrico. Esses processos podem causar um grande número se efeitos clínicos e eles são subdivididos de acordo com as suas manifestações clínicas. Nos efeitos fotoquímicos podemos citar abioestimulação, que se refere ao efeito da luz laser sobre processos moleculares e bioquímicos que normalmente ocorrem nos tecidos, como, por exemplo, a cicatrização de feridas e o reparo ósseo [16].

LED é a sigla, em inglês, para Light EmittingDiode, que em português significa diodo emissor de luz.

O aumento na deposição de colágeno após a irradiação com LED foi documentado em culturas de fibroblastos e em modelos humanos nos quais foi observada também a diminuição da colagenase [18] na cicatrização tecidual em modelos de queimadura de terceiro grau cicatrizante e em lesões bolhosas humanas18. Há evidências de que a luz produzida por LEDs, nos mesmos comprimentos de ondas bioestimulatórios de estudos anteriores com o laser, tem efeitos bioquímicos similares [17].

A fototerapia é uma modalidade terapêutica que possui atividade antiinflamatória, que promove a regeneração e o reparo dos tecidos conjuntivos por meio da estimulação da síntese de matriz extracelular e da proliferação celular [19], e o aumento da atividade de osteoblastos, vascularização e maior organização nas fibras colágenas após a incidência de irradiação luminosa sobre os tecidos [21].

A terapia com laser em baixa intensidade (LILT) vem sendo utilizada em diversas áreas da saúde com o objetivo de promover aceleração na regeneração tecidual, modulação de efeitos inflamatórios e erradicação ou redução de processos de dor[5,19]. Estes efeitos estão associados com a estimulação da microcirculação, efeitos antioxidantes, e reativação do potencial respiratório celular promovidos pela radiação laser em baixa intensidade, auxilia na cicatrização com por meio dos seus efeitos biomodulares [19, 21,22].

Os lasers de baixa intensidade foram os percussores na fototerapia para úlceras e nos últimos anos, com o avanço da microeletrônica, conseguiu-se criar pequenos diodos emissores de luz (LEDs ou LED T- LED Terapêutico) capazes de emitir grandes intensidades de luz, produzindo uma banda de espectro eletromagnético próxima do laser e uma consequente bioestimulação das células, intitulada ledterapia.

O tratamento com laser é um método rápido e eficaz, não tem contraindicações, e pode ser feito em conjunto com qualquer outro método de tratamento. 
Artigo de Revisão

Hegemonia - Revista Eletrônica do Programa de Mestrado em Direitos Humanos,

Cidadania e Violência/Ciência Política do Centro Universitário Unieuro

ISSN: 1809-1261

UNIEURO, Brasília, número 27 (Especial), 2019, pp. 7-27.

O número de sessões é variável de acordo com o tipo e tamanho da ferida e as sessões podem ser frequentes [23].

Uma importante indicação clínica da ledterapia como ferramenta terapêutica complementar é sua utilização no processo de cicatrização de feridas crônicas vasculares diabéticas e úlceras de pressão (escaras), além de ajudar em todas as feridas cirúrgicas em pós-operatórios [23].

Por não possuir contraindicações a ledterapia poder utilizada em conjunto com qualquer outro método de tratamento como, por exemplo, coberturas (curativos) que auxiliem também no processo cicatricial [23].

Portadores de Diabetes mellitus (DM) apresenta várias alterações no processo cicatricial, devido a essa dificuldade de cicatrização existe uma constante procura por novos tipos de tecnologias que possibilitem a manutenção da qualidade da pele e auxiliem a aceleração do processo cicatricial.

Nteleki e Houreld em um artigo de revisão aborda que a fototerapia (laserterapia de baixa intensidade - LLLT) é uma modalidade terapêutica que pode melhorar a cicatrização de feridas [24].

A literatura mostra que algumas pesquisas estudaram os efeitos do LED na regeneração tecidual, os resultados foram bem relevantes. $O$ trabalho o objetivo era analisar os resultados da aplicação da luz emitida pelos LEDs de alto brilho sobre o tecido humano. A aplicação obteve êxito na terapia, apresentando ótimos resultados nos pacientes que fizeram aledterapia [25, 26]. Já em [27] avaliou-se a fototerapia na cicatrização de úlceras de perna mistas em dois pacientes diabéticos (tipo 2) hipertensos. O aparelho apresentava sonda 1 (1 LED de 660nm, 5mW) aplicado em três úlceras e sonda 2 (32 LEDs de 890nm e 4 LEDs de $660 \mathrm{~nm}, 500 \mathrm{~mW}$ ) em seis úlceras. Após antissepsia, úlceras foram tratadas com sondas a $3 \mathrm{~J} / \mathrm{cm}^{2}, 30 \mathrm{seg}, 2 \mathrm{x} /$ semana seguido pelo curativo diário comsulfadiazina de prata a 1\%. A fototerapia acelerou a cicatrização das úlceras de perna em pacientes diabéticos [25].

Em outra pesquisa foi verificado a eficácia de um protótipo de fotobiomodulação constituído de LEDs, na faixa espectral do infravermelho próximo, no tratamento dos traumas mamilares. Dez participantes com 19 lesões mamilares (rachaduras, fissuras e escoriações) foram divididas em dois grupos: experimental e controle [28, 29].

Os resultados deste estudo mostraram que o protótipo de fotobiomodulação constituído com LEDs na faixa espectral do infravermelho próximo foi um recurso eficaz no tratamento dos traumas mamilares. 
Artigo de Revisão

Hegemonia - Revista Eletrônica do Programa de Mestrado em Direitos Humanos,

Cidadania e Violência/Ciência Política do Centro Universitário Unieuro

ISSN: 1809-1261

UNIEURO, Brasília, número 27 (Especial), 2019, pp. 7-27.

Um método inovador de regeneração tecidual de úlceras diabéticas foi desenvolvido, sendo ele composto pela ação conjunta e simultânea do biomaterial látex e da irradiação de luz de LEDs de baixa intensidade [25, 29]. Esse circuito eletrônico de radiação da luz de LED tem um temporizador para controlar o tempo de emissão da luz e um sonorizador para indicar o término da emissão. As células de irradiação da luz, inseridas externamente a palmilha e cobertas com uma lâmina de látex, emite uma irradiação com fluência de $25 \mathrm{~J} / \mathrm{cm}^{2}$. O estudo foi conduzido nas dependências do Hospital Regional de Taguatinga (HRT), localizado em Taguatinga - Distrito Federal, e os resultados foram bastante satisfatórios $[25,29]$.

\section{SISTEMA INDUTOR DE NEOFORMAÇÃO TECIDUAL - RAPHA}

Atualmente tem se desenvolvido uma nova possibilidade para tratar o pé diabético, induzindo assim a cicatrização das úlceras de pés diabéticos - UPD [30].

O projeto RAPHA - Equipamento Médico Portátil de Neoformação Tecidual (TC 129/20170) foi desenvolvido na UnB, e testado em humanos em hospitais em parceria ao GDF, mostrando ser bastante eficaz e um dos objetivos e incorporar essa tecnologia ao Sistema Único de Saúde (SUS) [30].

O Sistema Indutor de Neoformação tecidual é um aparelho para tratamento de pés diabéticos, que possui um circuito emissor de luz de LEDs, composto por duas placas de circuito impresso sendo uma placa de controle e uma placa de irradiação luminosa, associado com lâminas de látex natural, que estimula o processo cicatricial de lesões abertas $[24,28]$.

O RAPHA, uma palavra hebraica que significa cura é um aparelho portátil de fácil uso onde o próprio paciente faz a aplicação e pode ser monitorado continuamente por um profissional através de um aplicativo de monitoração [30].

Esse sistema possuir custos reduzidos devido à utilização de LEDs de alto brilho no lugar do LASER, acelerando o tratamento de pacientes, além do mais e de fácil aplicabilidade [30].

Esse equipamento e usando juntamente com uma membrana de látex natural extraído da seringueira brasileira (Hevea brasiliensis) é um sistema polidisperso, possui propriedades indutoras de neovascularização e regeneração tecidual, e formação de matriz extracelular, comprovada em várias espécies e em diferentes estudos do organismo [31, 32]. 
Artigo de Revisão

Hegemonia - Revista Eletrônica do Programa de Mestrado em Direitos Humanos,

Cidadania e Violência/Ciência Política do Centro Universitário Unieuro

ISSN: 1809-1261

UNIEURO, Brasília, número 27 (Especial), 2019, pp. 7-27.

O látex tem se destacado por ser um material com alto potencial de regeneração e apresenta diversas aplicações biomédicas para uso em próteses e enxertos médicos graças à sua biocompatibilidade, e por isso, é usado como suporte para a liberação sustentada de diferentes compostos (como fármacos, extratos, vegetais, moléculas, nanopartículas, proteínas e fosfato de cálcio) [33].

Estudos confirma que a biomembrana de látex age como agente indutor angiogênico, de alta qualidade e baixo custo quando comparado aos outros tipos de tratamentos convencionais, e uma boa opção terapêutica devido às diversas propriedades que possui [34].

A biomembrana de látex, já vem sido estudada, mostrando, o poder cicatrizante que possui, em pacientes crônicos e portadores de diabetes, em alguns casos deu a entender que a liberação de uma substância parecida com VEGFC (Fator de Crescimento Vasculo - Endotelial) e dessa forma reconstruindo veias e artérias, levando assim nutrientes para o local da lesão [34].

Tanto o equipamento quanto a biomembrana de látex ambos agentes possuem características e propriedades capazes de induzir a regeneração e neoformação tecidual.

\section{METODOLOGIA}

Foi realizada uma revisão de literatura científica utilizando os bancos de dados Scielo, Lilacs, PubMed, Bireme, Medline. Após a definição dessas bases de dados, foram definidos os descritores e os critérios de inclusão e de exclusão de artigos.

Para o estudo foram consideradas publicações entre o período de 2009 a 2018, com os seguintes descritores: "diabetes mellitus", "pé diabético”, "processo de cicatrização tecidual", "fototerapia", "RAPHA".

Com o intuito de realizar busca bibliográfica de artigos mais abrangente e relevante foram utilizados os termos combinados ou isolados nos idiomas inglês e português.

Foram estabelecidos os seguintes critérios de inclusão para a seleção de publicações: publicações completas; publicados e indexados nas bases de dados citadas no período de 2009 a 2018; e de acesso livre. 
Artigo de Revisão

Hegemonia - Revista Eletrônica do Programa de Mestrado em Direitos Humanos,

Cidadania e Violência/Ciência Política do Centro Universitário Unieuro

ISSN: 1809-1261

UNIEURO, Brasília, número 27 (Especial), 2019, pp. 7-27.

Como critérios de exclusão os artigos publicados antes de 2008; que não possuíam resumo; publicações que não estavam relacionando à fototerapia como tratamento de cicatrização.

Foi empregada a revisão sistemática por permitir evidências do uso de fototerapia na cicatrização de lesões em pés diabéticos. Dessa maneira, contribuindo para soluções eficazes e mais apropriadas para cada situação.

\section{RESULTADOS}

A pesquisa foi realizada no segundo bimestre do ano de 2018. Foi encontrado um total de 637 artigos a partir dos descritores selecionados. Apenas 48 apresentaram-se potencialmente consideráveis para o estudo. Foram retiradas as duplicidades de publicações, artigos estavam presentes mais de uma base de dados.

Após uma análise mais minuciosa foram excluídos 21 artigos, portanto sendo selecionados 27 para avaliação detalhada. Assim, para uma avaliação mais criteriosa foram recuperadas as publicações completas.

Foram excluídos os artigos que não apresentavam os requisitos mínimos exigidos para este estudo. E 11 artigos atenderam os critérios de inclusão.

A tabela 1 retrata os resultados da revisão de literatura científica, e a caracterizações desses quanto ao autor, ano, estudo, objetivos, metodologia e conclusões. 
Artigo de Revisão

Hegemonia - Revista Eletrônica do Programa de Mestrado em Direitos Humanos, Cidadania e Violência/Ciência Política do Centro Universitário Unieuro

ISSN: 1809-1261

UNIEURO, Brasília, número 27 (Especial), 2019, pp. 7-27. 
Artigo de Revisão

Hegemonia - Revista Eletrônica do Programa de Mestrado em Direitos Humanos, Cidadania e Violência/Ciência Política do Centro Universitário

Unieuro

ISSN: 1809-1261

UNIEURO, Brasília, número 27 (Especial), 2019, pp. 7-27.

TABELA I

PUBLICACÕES PESQUISADAS

\begin{tabular}{|c|c|c|c|c|c|c|}
\hline & Autor & Ano & Estudo & Objetivos & Metodologia & PrincipaisConclusões \\
\hline 1 & $\begin{array}{l}\text { H.T. } \\
\text { Wang et } \\
\text { al. }\end{array}$ & 2017 & $\begin{array}{l}\text { Phototherapy for treating } \\
\text { foot ulcers in people with } \\
\text { diabetes }\end{array}$ & $\begin{array}{l}\text { Avaliar os efeitos da fototerapia para o } \\
\text { tratamento de úlceras nos pés em pessoas } \\
\text { com diabetes }\end{array}$ & $\begin{array}{l}\text { Revisão sistemática de } \\
\text { estudos randomizados }\end{array}$ & $\begin{array}{l}\text { A fototerapia, quando comparada a nenhuma } \\
\text { fototerapia / placebo, pode aumentar a } \\
\text { proporção de feridas completamente } \\
\text { cicatrizadas durante o acompanhamento e pode } \\
\text { reduzir o tamanho da ferida em pessoas com } \\
\text { diabetes. }\end{array}$ \\
\hline 2 & $\begin{array}{l}\text { G.A.M. } \\
\text { A. } \\
\text { Nunes } \\
\text { et al }\end{array}$ & 2016 & $\begin{array}{l}\text { A system for treatment of } \\
\text { diabetic foot ulcers using } \\
\text { led irradiation and natural } \\
\text { latex }\end{array}$ & $\begin{array}{l}\text { Desenvolver e testar um sistema para } \\
\text { induzir a regeneração de úlceras do pé } \\
\text { diabético com base em uma palmilha de } \\
\text { látex natural e um dispositivo usando } \\
\text { diodos emissores de luz (LEDs) }\end{array}$ & Estudo clínico & $\begin{array}{l}\text { Os resultados sugerem que o método proposto } \\
\text { parece promissor como um método de } \\
\text { tratamento futuro. No entanto, a técnica deve } \\
\text { passar por mais testes antes que possa ser } \\
\text { considerada para extensas aplicações clínicas. }\end{array}$ \\
\hline 3 & $\begin{array}{l}\text { A.F.M. } \\
\text { Carvalh } \\
\text { o et al. }\end{array}$ & 2016 & $\begin{array}{l}\text { Low-level laser therapy and } \\
\text { Calendula officinalis in } \\
\text { repairing diabetic foot } \\
\text { ulcers }\end{array}$ & $\begin{array}{l}\text { Avaliar os efeitos da Terapia a Laser de } \\
\text { Baixa Intensidade isolada e associada ao } \\
\text { óleo de Calendulaofficinalis no reparo de } \\
\text { úlceras em pé diabético }\end{array}$ & $\begin{array}{l}\text { Estudo de caso } \\
\text { clínico, experimental, } \\
\text { controlado, } \\
\text { randomizado, } \\
\text { prospectivo, } \\
\text { intervencional, de } \\
\text { caráter quantitativo }\end{array}$ & $\begin{array}{l}\text { A Terapia a Laser de Baixa Intensidade, } \\
\text { realizada tanto isoladamente quanto associada } \\
\text { ao óleo de Calendulaofficinalis, foi eficaz no alívio } \\
\text { da dor e na aceleração do processo de reparo } \\
\text { tecidual de pé diabético. }\end{array}$ \\
\hline 4 & $\begin{array}{l}\text { R.G. } \\
\text { Sousa } \\
\text { and } \\
\text { K.N.M. } \\
\text { Batista }\end{array}$ & 2016 & $\begin{array}{l}\text { Laser therapy in wound } \\
\text { healing associated with } \\
\text { diabetes mellitus - Review. }\end{array}$ & $\begin{array}{l}\text { Determinar o parâmetro mais eficaz na } \\
\text { cicatrização de feridas relacionadas ao } \\
\text { diabetes mellitus, bem como o tipo de } \\
\text { laser mais utilizado }\end{array}$ & Revisão sistemática & $\begin{array}{l}\text { Os melhores resultados na cicatrização de } \\
\text { feridas diabéticas foram aqueles que aplicaram } \\
\text { densidades de energia na faixa de } 3-5 \mathrm{~J} / \mathrm{cm} 2 \text {, } \\
\text { densidades de potência } \leq 0,2 \mathrm{~W} / \mathrm{cm} 2 \text { e } \\
\text { emissão contínua. O laser He-Ne com } \\
\text { comprimento de onda de } 632,8 \mathrm{~nm} \text { foi o mais } \\
\text { utilizado }\end{array}$ \\
\hline
\end{tabular}


Artigo de Revisão

Hegemonia - Revista Eletrônica do Programa de Mestrado em Direitos Humanos, Cidadania e Violência/Ciência Política do Centro Universitário Unieuro

ISSN: 1809-1261

UNIEURO, Brasília, número 27 (Especial), 2019, pp. 7-27. 
Artigo de Revisão

Hegemonia - Revista Eletrônica do Programa de Mestrado em Direitos Humanos, Cidadania e Violência/Ciência Política do Centro Universitário

Unieuro

ISSN: 1809-1261

UNIEURO, Brasília, número 27 (Especial), 2019, pp. 7-27.

TABELA I

PUBLICAÇÕESPESQUISADAS

\begin{tabular}{|c|c|c|c|c|c|c|}
\hline & Autor & Ano & Estudo & Objetivos & Metodologia & PrincipaisConclusões \\
\hline 5 & $\begin{array}{l}\text { C.N. } \\
\text { Tchanque- } \\
\text { Fossuo et al. }\end{array}$ & 2016 & $\begin{array}{l}\text { A systematic review of low- } \\
\text { level light therapy for } \\
\text { treatment of diabetic foot } \\
\text { ulcer }\end{array}$ & $\begin{array}{l}\text { Examinar se o uso clínico de terapia de luz } \\
\text { de baixo nível é eficaz na cicatrização de } \\
\text { úlceras do pé diabético em } 12 \text { e } 20 \\
\text { semanas, em comparação com o padrão de } \\
\text { atendimento, e fornecer recomendações } \\
\text { baseadas em evidências e futuras diretrizes } \\
\text { clínicas para o tratamento dessas úlceras } \\
\text { usando a fototerapia mencionada. }\end{array}$ & Revisão sistemática & $\begin{array}{l}\text { A terapia de luz de baixo nível tem um potencial } \\
\text { significativo para se tornar uma modalidade } \\
\text { portátil, minimamente invasiva, fácil de usar e } \\
\text { econômica para o tratamento de úlceras de pé } \\
\text { diabético. São necessários estudos adicionais } \\
\text { com parâmetros de laser comparáveis }\end{array}$ \\
\hline 6 & $\begin{array}{l}\text { R.G. Sousa } \\
\text { and K.N.M. } \\
\text { Batista }\end{array}$ & 2016 & $\begin{array}{l}\text { Laser therapy in wound } \\
\text { healing associated with } \\
\text { diabetes mellitus - Review }\end{array}$ & $\begin{array}{l}\text { Determinar o parâmetro mais eficaz na } \\
\text { cicatrização de feridas relacionadas ao } \\
\text { diabetes mellitus, bem como o tipo de } \\
\text { laser mais utilizado }\end{array}$ & Revisão sistemática & $\begin{array}{l}\text { Os estudos que mostraram resultados mais } \\
\text { satisfatórios na cicatrização de feridas diabéticas } \\
\text { foram aqueles que aplicaram densidades de } \\
\text { energia na faixa de } 3-5 \mathrm{~J} / \mathrm{cm} 2 \text {, densidades de } \\
\text { potência iguais ou inferiores a } 0,2 \mathrm{~W} / \mathrm{cm} 2 \text { e } \\
\text { emissão contínua. O laser He-Ne com } \\
\text { comprimento de onda de } 632,8 \mathrm{~nm} \text { foi utilizado } \\
\text { com maior frequência }\end{array}$ \\
\hline 7 & $\begin{array}{l}\text { M.C.P. } \\
\text { Feitosa et al. }\end{array}$ & 2015 & $\begin{array}{l}\text { Effects of the Low-Level } \\
\text { Laser Therapy (LLLT) in } \\
\text { the process } \\
\text { of healing diabetic } \\
\text { foot ulcers }\end{array}$ & $\begin{array}{l}\text { Avaliar os efeitos da laserterapia de baixa } \\
\text { intensidade com laser no reparo tecidual } \\
\text { em portadores de úlcera por diabetes }\end{array}$ & $\begin{array}{l}\text { Estudo de caso } \\
\text { clínico, controlado, } \\
\text { randomizado, } \\
\text { intervencionista, de } \\
\text { caráter quantitativo }\end{array}$ & $\begin{array}{l}\text { O tratamento com laser de baixa intensidade } \\
\text { parece ser um método eficiente, viável, indolor } \\
\text { e de baixo custo para as úlceras de reparo } \\
\text { tecidual em pé diabético }\end{array}$ \\
\hline 8 & $\begin{array}{l}\text { N.N. } \\
\text { Houreld }\end{array}$ & 2014 & $\begin{array}{l}\text { Shedding light on a new } \\
\text { treatment for diabetic } \\
\text { wound healing: a review } \\
\text { on phototherapy }\end{array}$ & $\begin{array}{l}\text { Analisar as complicações associadas à } \\
\text { cicatrização de feridas diabéticas e o efeito } \\
\text { da irradiação a laser tanto in vitro quanto } \\
\text { in vivo na cicatrização de feridas } \\
\text { diabéticas. }\end{array}$ & Revisão sistemática & $\begin{array}{l}\text { A fototerapia tem demonstrado ser benéfica no } \\
\text { tratamento de úlceras diabéticas que não } \\
\text { respondem aos tratamentos convencionais }\end{array}$ \\
\hline
\end{tabular}


Artigo de Revisão

Hegemonia - Revista Eletrônica do Programa de Mestrado em Direitos Humanos, Cidadania e Violência/Ciência Política do Centro Universitário

Unieuro

ISSN: 1809-1261

UNIEURO, Brasília, número 27 (Especial), 2019, pp. 7-27.

TABELA I

PUBLICACÕES PESQUISADAS

\begin{tabular}{|c|c|c|c|c|c|c|}
\hline & Autor & Ano & Estudo & Objetivos & Metodologia & Principais Conclusões \\
\hline 9 & $\begin{array}{l}\text { K.H. } \\
\text { Beckmann, } \\
\text { G.Meyer- } \\
\text { Hamme and } \\
\text { S. Schröder }\end{array}$ & 2014 & $\begin{array}{l}\text { Low level laser therapy for } \\
\text { the treatment of diabetic } \\
\text { foot ulcers: a critical survey }\end{array}$ & $\begin{array}{l}\text { Obter evidência conclusiva de laser de } \\
\text { baixo nível no tratamento de diabetes } \\
\text { úlceras do pé }\end{array}$ & Revisão & $\begin{array}{l}\text { Há evidências suficientes para continuar a } \\
\text { pesquisa sobre a terapia a laser para úlceras } \\
\text { diabéticas, mas ensaios clínicos usando modelos } \\
\text { humanos não fornecem evidências suficientes } \\
\text { para estabelecer eficácia da terapia de laser de } \\
\text { baixo nível. }\end{array}$ \\
\hline 10 & $\begin{array}{l}\text { B.M. Kajagar } \\
\text { et al. }\end{array}$ & 2012 & $\begin{array}{l}\text { Efficacy of low level laser } \\
\text { therapy on wound healing } \\
\text { in patientswith chronic } \\
\text { diabetic foot ulcers - a } \\
\text { randomized control trial }\end{array}$ & $\begin{array}{l}\text { Avaliar a eficácia da Terapia a Laser de } \\
\text { Baixo Nível (LLLT) na dinâmica da } \\
\text { cicatrização de úlceras diabéticas. }\end{array}$ & $\begin{array}{l}\text { Ensaio clínico } \\
\text { randomizado }\end{array}$ & $\begin{array}{l}\text { A terapia a laser de baixo nível é benéfica como } \\
\text { adjuvante as terapias convencionais no } \\
\text { tratamento de úlceras do pé diabético }\end{array}$ \\
\hline 11 & $\begin{array}{l}\text { M.V.M. } \\
\text { Pinto et al. }\end{array}$ & 2012 & $\begin{array}{l}\text { Influência da laserterapia de } \\
\text { 632,8NM na cicatrização } \\
\text { diabética }\end{array}$ & $\begin{array}{l}\text { Verificar a influência da laserterapia AsGa } \\
(632,8 \mathrm{~nm}) \text {, com dose de } 120 \mathrm{~J} / \mathrm{cm}^{2} \text { de } \\
\text { potência na cicatrização diabética, } \\
\text { apresentando úlcera na região do calcâneo } \\
\text { e no hálux }\end{array}$ & Ensaio clínico & $\begin{array}{l}\text { O laser (AsGa) nestes parâmetros teve uma } \\
\text { ótima influência no tratamento de úlcera em pé } \\
\text { diabético no aspecto dor e cicatrização, não } \\
\text { importando a sua localização }\end{array}$ \\
\hline
\end{tabular}


Artigo de Revisão

Hegemonia - Revista Eletrônica do Programa de Mestrado em Direitos Humanos,

Cidadania e Violência/Ciência Política do Centro Universitário Unieuro

ISSN: $1809-1261$

UNIEURO, Brasília, número 27 (Especial), 2019, pp. 7-27.

\section{REFERÊNCIAS}

[1] Associação Geofilosófica de Estudos Antropológicos e Culturais: Papiro Ebers.

Disponível em: http://ageac.org/pt/noticias/o-papiro-ebers .Acessoem : 19/06/2018.

[2] BORTOLETTO, M.S.S. et al. Pé diabético, uma avaliação sistematizada. Arquivo de Ciências da Saúde Unipar, v.13, n.1, p.37-43, 2009.

[3] Sociedade Brasileira de Endocrinologia. Diabetes, 2010. Disponível em: https://www.endocrino.org.br/pe-diabetico/. Acesso em : 19/06/2018.

[4] PARISI, M.C.R. A síndrome do pé diabético, fisiopatologia e aspectos práticos. E-book 2.0 - Diabetes na prática Clínica (s.d.). Sociedade Brasileira de Diabetes. Disponívelem: https://www.diabetes.org.br/ebook/component/k2/item/42-asindrome-do-pe-diabetico-fisiopatologia-e-aspectos-praticos .Acessoem: 20/062018

[5] K.B.V. Dourado.; J.L.C. Carnevali.; R.J.F. Paulo and A.C. Gomes. Ledterapia: Uma nova perspectiva terapêutica ao tratamento de doenças da pele, cicatrização de feridas e reparação tecidual. Ensaios e Ciência: Ciências Agrárias Biológicas e Da Saude, v.15,n.6, p. 231-248, 2011.

[6] M.H.M. Lima and E.P. Araújo. "Diabetes Mellitus andtheprocessofcutaneoushealing" Revista Cogitare Enfermagem, vol.18, no.1 pp.170-2. Rev.CogitareEnferm Jan/Mar,2013.

[7] G.A. Nuneset al. "A system for treatment of diabetic foot ulcers using led irradiation and natural latex" Research on Biomedical Engineering, vol.32, no.1 pp.3-13. Res. Biomed EngFeb, 2016. http://dx.doi.org/10.1590/2446-4740.0744.

[8] M.S. Panobiancoet al. "Comparação da cicatrização pós-mastectomia entre mulheres portadoras e não-portadoras de diabetes mellitus"Revista Rene, vol.11, no. especial pp.15-22. Rev. Rene Dez, 2012

[9] N. Duarte and A. Gonçalves. "Diabeticfoot" Angiologia e Cirurgia Vascular, vol.7, no.2 pp.65-7. Ang e Cirur Vasc. Junho, 2011.

[10] I.S. Martin et al "Causas referidas para o desenvolvimento de úlceras em pés de pessoas com diabetes mellitus.”Acta Paul Enferm, São Paulo, v.25,n.2,p.218-224, 2012.

[11] C.A. Balbino et al. " Mecanismos envolvidos na cicatrização: uma revisão" Revista Brasileira de Ciências Farmacêuticas, vol.41, no.1 pp.27-51. Rev. Bras. Cienc. Farm. Jan/Mar, 2005. http://dx.doi.org/10.1590/S1516-93322005000100004.

[12] I.V.P.M. Oliveira and R.V.C. Dias. "Wound healing: stages and influencing factors" ActaVeterinariaBrasilica, vol.6, no.4 pp.267-271. Acta Vet Brasi, 2012. 
Artigo de Revisão

Hegemonia - Revista Eletrônica do Programa de Mestrado em Direitos Humanos,

Cidadania e Violência/Ciência Política do Centro Universitário Unieuro

ISSN: 1809-1261

UNIEURO, Brasília, número 27 (Especial), 2019, pp. 7-27.

[13] V. Kumaret al. Patologia - Bases Patológicas das Doenças. 8 ed. Rio de Janeiro: Elsevier; 2010

[14] E.A. Gantwerker and D.B.Hom. Skin: Histology and Physiology of Wound Healing. ClinPlasticSurg 2012; vol. 39 pp.85-97.

[15] R.J. Mendonça andJ.Coutinho-Neto. "Aspectos celulares da cicatrização" Anais Brasileiros de Dermatologia, vol. 84, no.3 pp.257-62.AnBrasDermatol Jan, 2009. http://producao.usp.br/handle/BDPI/7230

[16] A.L.B. Pinheiro, J. Brugnerar, F.A.A. Zanin. "Aplicação do Laser na Odontologia”. São Paulo: Santos. 2010;428.

[17] PINHEIRO, L. B. Antônio,ALMEIDA, F. Paulo F, SOARES, P. Luiz Guilherme; "Princípios fundamentais dos lasers e suas aplicações", p. 815 -894. In: RESENDE, Rodrigo Ribeiro. Biotecnologia Aplicada à Agro\&Indústria - Vol. 4.São Paulo: Blucher, 2017. ISBN: 9788521211150, DOI 10.5151/9788521211150-23

[18] D. Baroletet al. "Regulation of skin collagen metabolism in vitro using a pulsed $660 \mathrm{~nm}$ LED light source: clinical correlation with a singleblinded study". J InvestigDermatol. 2009;129(12):2751-9

[19] P.C. Chang et al. "Irradiationby light-emittingdiode light as anadjuncttofacilitatehialing of experimental periodontitis in vivo." J. Periodont Res, Singapore, vol.48, pp. 135-143, 2012.

[20] R. Prateset al. Fototerapia com laser em baixa intensidade em processos infecciosos agudos. Centro de Lasers e Aplicações, IPEN-CNEN, São Paulo, 2010

[21] L.H. Theodoroet al. "Effectiveness of the diode laser in the treatment of ligatureinduced periodontitis in rats: a histopathological, histometric, and immunohistochemical study”. Lasers Med Sci, vol. 30, no. 4, pp. 1209-18, May 2015.

[22] F. Oliveira et al. "Ação do laser terapêutico e do ultrassom na regeneração nervosa periférica; Actionoftherapeutic laser andultrasound in peripheralnerveregeneration." Acta Ortopédica Brasileira. 2012;20(2):98-103.

[23] Tratamento de Feridas com Laserterapia. ALLASER, 2015. Disponível em: http://www.allaser.com.br/tratamentos-de-feridas. Acessoem: 20/05/2018.

[24] B. Nteleki and N. N. Houreld (2012). "Review Article: The use of phototherapy in the treatment of diabetic ulcers". JEMDSA; 17(3): 128-132. 
Artigo de Revisão

Hegemonia - Revista Eletrônica do Programa de Mestrado em Direitos Humanos,

Cidadania e Violência/Ciência Política do Centro Universitário Unieuro

ISSN: $1809-1261$

UNIEURO, Brasília, número 27 (Especial), 2019, pp. 7-27.

[25] M. REIS. Sistema indutor de neoformação tecidual para pé diabético com circuito emissor de luz de leds e utilização do latex natural, DF: Departamento de Engenharia Elétrica, Universidade de Brasília, 2013.

[26] M. C. Moreira (2009). "Utilização de conversores eletrônicos que alimentam LEDs de alto brilho na aplicação em tecido humano e sua interação terapêutica". Tese de Doutorado, Departamento de Engenharia Elétrica, Universidade Federal de Santa Maria, Santa Maria, RS, 190p.

[27] D. G. Minatel, , C. S. Enwemeka, , S. C. França, M. A. C. Frade (2009). "Fototerapia (LEDs 660/890nm) no tratamento de úlceras de perna em pacientes diabéticos: estudo de caso". AnBrasDermatol. 84(3):279-83

[28] M. E. A. Chaves (2011). "Validação de um protótipo fotobiomodulador para tratamento de traumas mamilares". Dissertação de Mestrado, Departamento de Engenharia Mecânica, Universidade Federal de Minas Gerais, Belo Horizonte, 62.

[29] G.A.M.A. Nunes et al. "A sytem for treatment of foot ulcers using led irradiation and natural latex". Research on Biomedical Engineering, Rio de Janeiro, v. 32, n.1, p.3-13, jan./mar.2016. http://dx.doi.org/10.1590/2446-4740.0744

[30] UnbCiência. Projeto Rapha acelera cura para pé diabético. Dispónivelem:https://www.unbciencia.unb.br/exatas/68-engenharia-eletronica/539 projeto-rapha-acelera-cura-para-o-pe-diabetico

[31] M. A. C. Frade. M. A. C. et al. Curativo de biomembrana vegetal e hipersensibilidade.Anais Brasileiros de Dermatologia, v. 86, n.5, pp.885-891, out. 2011

[32] ANDRADE, T. A. M. F. Modificações teciduais e mecanismos de ação da fração F1 do látex da seringueira Hevea brasiliensis na cicatrização de úlceras cutâneas em ratos diabéticos.2012.Dissertação de Mestrado - Universidade de São Paulo - São Paulo, 2012.

[33] Unespciência. Aplicações biomédicasda membrana de latex natural. Disponívelem: http://unespciencia.com.br/2017/09/01/biomedicina-89/. Acessoem 19/06/2018

[34] R. V. Braun. Evidências na prática de cicatrização tecidual com biomembrana de látex. 2016. xvi, 70 f. il. Dissertação (Mestrado em Engenharia Biomédica)—Universidade de Brasília, Brasília, 2016

[35] H..T. Wang et al. "Phototherapy for treating foot ulcers in people with diabetes". Cochrane Database of Systematic Reviews 2017, Issue 6. Art. no.: CD011979. DOI: 10.1002/14651858.CD011979.pub2 
Artigo de Revisão

Hegemonia - Revista Eletrônica do Programa de Mestrado em Direitos Humanos,

Cidadania e Violência/Ciência Política do Centro Universitário Unieuro

ISSN: 1809-1261

UNIEURO, Brasília, número 27 (Especial), 2019, pp. 7-27.

[36] A.F.M. Carvalho et al. "Low-level laser therapy and Calendula officinalis in repairing diabetic foot ulcers". Rev. esc. enferm. USP 2016, vol.50, no.4, pp.628-634. http://dx.doi.org/10.1590/S0080-623420160000500013

[37] R.G. Sousa and K.N.M. Batista. "Laser therapy in wound healing associated with diabetes mellitus - Review”. An. Bras. Dermatol, 2016, vol.91, n.4 pp.489-493.

[38] C.N. Tchanque-Fossuoet al. "A systematic review of low-level light therapy for treatment of diabetic foot ulcer”. Wound Repair Regen. 2016 Mar; vol.24, no. 2 pp.418-26. doi: 10.1111/wrr.12399. Epub 2016 Mar 2.

[39] M.C.P. Feitosa et al. "Effects of the Low-Level Laser Therapy (LLLT) in the process of healing diabetic foot ulcers". Acta Cir. Bras. Dec, 2015, vol.30, no.12 pp.852-857. http://dx.doi.org/10.1590/S0102-865020150120000010

[40] N.N. Houreld. "Shedding light on a new treatment for diabetic wound healing: a review on phototherapy”. ScientificWorldJournal. e-collection Jan, 2014. 2014:398412. doi: $10.1155 / 2014 / 398412$.

[41] B.M. Kajagaret al."Efficacy of low level laser therapy on wound healing in patients with chronic diabetic foot ulcers-a randomised control trial”. Indian J Surg. Oct, 2012. vol.74 no.5 pp.359-63. doi: 10.1007/s12262-011-0393-4.

[42] K.H. Beckmann, G.Meyer-Hamme and S. Schröder. "Low level laser therapy for the treatment of diabetic foot ulcers: a critical survey". Evid Based Complement Alternat Med.Mar,2014;2014:626127. doi: 10.1155/2014/626127

[43] R.G. Sousa and K.N.M. Batista. Laser therapy in wound healing associated with diabetes mellitus - Review. An. Bras. Dermatol.2016, vol.91, n.4 pp.489493.http://dx.doi.org/10.1590/abd1806-4841.20163778

[44] V.M Pinto et al.. "Influência da laserterapia de 632,8nm na cicatrização diabética". Ciências Biológicas e da saúde, vol. 6, no. 2 pp. 25-29. Biol. \& Saúde, 2012. 